\title{
Non-Perturbative Renormalization of Nucleon Charges with Automated Perturbative Subtraction
}

\author{
M. Hansen ${ }^{1}$, T. Harris ${ }^{1}$, G. von Hippel ${ }^{* 2}$, P. Junnarkar ${ }^{1}$, H. Wittig ${ }^{1,2}$, L. Wrang ${ }^{2,3}$ \\ ${ }^{1}$ Helmholtz-Institut Mainz, 55099 Mainz, Germany \\ ${ }^{2}$ Institut für Kernphysik, Johannes Gutenberg-Universität Mainz, 55099 Mainz, Germany \\ ${ }^{3}$ University of Uppsala, Uppsala, Sweden \\ E-mail: hippel@kph.uni-mainz.de
}

\begin{abstract}
We report on the determination of the renormalization factors of quark bilinears which are required among others in order to determine the nucleon scalar and tensor charges from the CLS $N_{\mathrm{f}}=2$ configurations. Working in the RI'-MOM scheme, we eliminate all lattice artifacts at one-loop order using a combination of analytical results near the continuum limit and numerical calculations in automated lattice perturbation theory. The latter will allow for a ready generalization to the renormalization factors required for the average momentum fraction and other operators beyond local bilinears.
\end{abstract}

34th annual International Symposium on Lattice Field Theory

24-30 July 2016

University of Southampton, UK

${ }^{*}$ Speaker. 


\section{Introduction}

The axial, scalar and tensor charges of the nucleon (and the associated form factors) encode important information about nucleon structure. As in particular the scalar and tensor charges are hard to probe experimentally, good theoretical predictions are needed.

Since the charges are matrix elements of composite operators, they need to be renormalized, and it has long been known that perturbative renormalization may not be sufficient; nonperturbative renormalization (NPR) is required, but elaborate techniques must be applied to obtain reliable estimates. This is even more relevant for matrix elements of derivative operators, such as are needed to obtain the average momentum fraction $\langle x\rangle$ and related observables. Here, we demonstrate the usefulness of automated lattice perturbation theory in subtracting lattice artifacts using quark bilinears (whose matrix elements yield the nucleon charges) as a straightforward example.

\section{NPR setup}

We use the RI'-MOM scheme [1] in Landau gauge, with renormalization conditions

$$
\begin{array}{r}
\left.\operatorname{tr}_{C D}\left[S_{R}^{-1}(p) S_{\text {free }}(p)\right]\right|_{p^{2}=\mu^{2}}=12, \\
\left.\operatorname{tr}_{C D}\left[\left\langle p\left|O_{R}\right| p\right\rangle\left\langle p\left|O_{0}\right| p\right\rangle_{\text {free }}^{-1}\right]\right|_{p^{2}=\mu^{2}}=12 .
\end{array}
$$

Under the assumption that fields and operators renormalize multiplicatively, $S_{R}(p)=Z_{q} S_{0}(p)$, $O_{R}=Z_{O} O_{0}$, these conditions imply that the renormalization factors are given by

$$
\begin{aligned}
& Z_{q}=\left.\frac{1}{12} \operatorname{tr}_{C D}\left[S_{0}^{-1}(p) S_{\text {free }}(p)\right]\right|_{p^{2}=\mu^{2}}, \\
& Z_{O}=\frac{12 Z_{q}}{\left.\operatorname{tr}_{C D}\left[\Lambda_{O}(p) \Lambda_{O}^{\text {free }}(p)^{-1}\right]\right|_{p^{2}=\mu^{2}}},
\end{aligned}
$$

where the bare vertex function $\Lambda_{O}$ is given in terms of the bare Green's functions $G_{O}$ and $S_{0}$ through

$$
\Lambda_{O}(p)=S_{0}^{-1}(p) G_{O}(p) S_{0}^{-1}(p) .
$$

The latter are determined using momentum sources [2] to compute position-momentum propagators $S(y \mid p)=D_{y x}^{-1} \mathrm{e}^{i p \cdot x}$, whence

$$
S_{0}(p)=\left\langle\frac{1}{V} \sum_{x} \mathrm{e}^{-i p \cdot x} S(x \mid p)\right\rangle
$$

and for a local bilinear operator $O(x)=\bar{u}(x) \Gamma_{O} d(x)$,

$$
G_{O}(p)=\left\langle\frac{1}{V} \sum_{x} \gamma_{5} S(x \mid p)^{\dagger} \gamma_{5} \Gamma_{O} S(x \mid p)\right\rangle .
$$

We employ the $N_{\mathrm{f}}=2$ CLS ensembles listed in Table 1, using $N=20$ configurations from each ensemble, which we fix to Landau gauge by minimizing

$$
W(U)=\sum_{x} \sum_{\mu} \operatorname{tr}\left[U_{\mu}^{\dagger}(x)+U_{\mu}(x)\right]
$$

using a Fourier-accelerated CG algorithm [3]. 


\begin{tabular}{ccccc}
\hline Name & $\beta$ & $a[\mathrm{fm}]$ & Volume & $m_{\pi}[\mathrm{MeV}]$ \\
\hline A3 & 5.2 & 0.0755 & $64 \times 32^{3}$ & 473 \\
A4 & & & & 364 \\
A5 & & & & 316 \\
B6 & & & $96 \times 48^{3}$ & 268 \\
\hline E5 & 5.3 & 0.0658 & $64 \times 32^{3}$ & 457 \\
F6 & & & $96 \times 48^{3}$ & 324 \\
F7 & & & & 277 \\
G8 & & & $128 \times 64^{3}$ & 193 \\
\hline N5 & 5.5 & 0.0486 & $96 \times 48^{3}$ & 429 \\
N6 & & & & 331 \\
O7 & & & $128 \times 64^{3}$ & 261 \\
\hline
\end{tabular}

Table 1: List of CLS ensembles used in this study, giving the ensemble name, bare inverse coupling $\beta=$ $6 / g_{0}^{2}$, lattice spacing, spacetime volume and pion mass.

To reduce $\mathrm{O}(4)$ violation effects, we use diagonal momenta of the form $p=(\mu, \mu, \mu, \mu)$, where twisted boundary conditions $\psi\left(x+L_{v} e_{v}\right)=\mathrm{e}^{i \theta_{v}} \psi(x)$ are employed to allow access to intermediate momenta. A further reduction of $\mathrm{O}(4)$ violating effects is achieved by averaging over $\mathrm{H}(4)$ irreps $\ell$ [4], corresponding to replacing

$$
\operatorname{tr}_{C D}\left[\Lambda_{O}(p) \Lambda_{O}^{\mathrm{free}}(p)\right] \mapsto \frac{1}{K} \sum_{\ell=1}^{K} \operatorname{tr}_{C D}\left[\Lambda_{O}^{\ell}(p) \Lambda_{O}^{\ell \text {,free }}(p)\right]
$$

in eq. (2.4).

Given the values of the renormalization constants at our chosen momenta, we interpolate in $\mu$ on each ensemble, using cubic splines, in order to get $Z\left(a, m_{\pi} ; \mu\right)$ for arbitrary renormalization scales $\mu$, which we then use to chirally extrapolate for a set of values of $\mu$ at each value of $\beta$, using a linear ansatz in $\left(a m_{\pi}\right)^{2}$, to obtain $Z(a, \mu)=Z(a, 0 ; \mu)$.
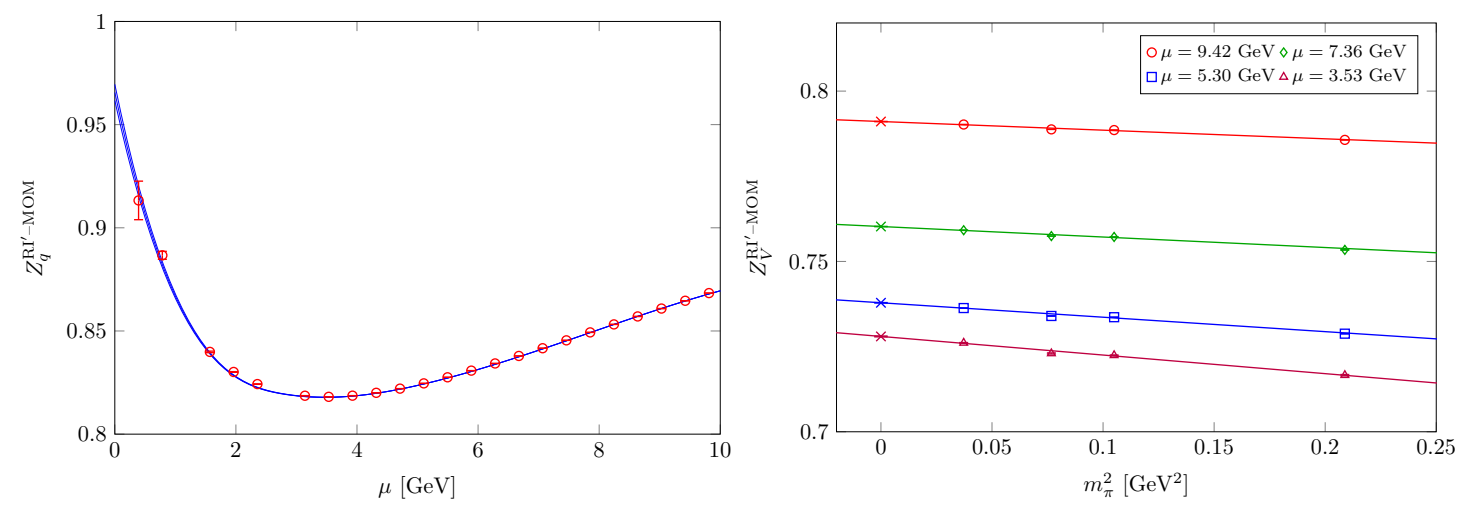

Figure 1: left: example of cubic-spline interpolation in $\mu$; right: example of the chiral extrapolation. 
To convert from the RI'-MOM scheme to the more usual $\overline{\mathrm{MS}}$-scheme, we use the threeloop continuum perturbation theory results of [5]. In addition, we determine the Renormalization Group Independent (RGI) values of the renormalization factors using the three-loop $\overline{\mathrm{MS}} \beta$ - and $\gamma$-functions [6] in

$$
Z^{\mathrm{RGI}}(a)=\Delta Z^{\overline{\mathrm{MS}}}(\mu) Z_{\mathrm{RI}^{\prime}-\mathrm{MOM}}^{\overline{\mathrm{MS}}}(\mu) Z^{\mathrm{RI}^{\prime}-\mathrm{MOM}}(a, \mu) .
$$

\section{Perturbative subtraction}

By definition, the RGI renormalization factors should be independent of the renormalization scale $\mu$. Since the running is only removed perturbatively, we expect deviations at small $\mu$, where the running coupling becomes large. On the other hand, any $\mu$-dependence at large $\mu$, where the running coupling is small, is indicative of lattice artifacts, which in practice can be quite sizeable.

To eliminate at least a large portion of lattice artifacts, the use of perturbation theory has been proposed in ref. [7], and further explored in refs. [8, 9]; here we follow an approach very similar to that of ref. [4], subtracting all lattice artifacts at $\mathrm{O}\left(g^{2}\right)$ by perturbatively expanding

$$
Z^{\mathrm{RI}^{\prime}-\mathrm{MOM}}(\mu, a)=1+g^{2} F(\mu, a)=1+g^{2}\left[\gamma_{0} \log (\mu a)+C+\mathrm{O}\left(\mu^{2} a^{2}\right)\right],
$$

where $\gamma_{0}$ is the analytically-known anomalous dimension. The lattice artifacts are then given by

$$
D(\mu, a)=g^{2}\left[F(\mu, a)-\left(\gamma_{0} \log (\mu a)+C\right)\right],
$$

where in many cases $C$ is known analytically, or else can be obtained using a fit to $F(\mu, a)-$ $\gamma_{0} \log (\mu a)$ in the limit $a \rightarrow 0$.

To evaluate the required Feynman diagrams in lattice perturbation theory, we employ the HiPPy/HPsrc packages [10], which separate the (complicated, action-dependent) Feynman rules from the (action-independent) Feynman diagrams: the diagrams are coded once and for all in an operator- and action-independent fashion, and can then be used to calculate with in principle arbitrary operators and lattice actions. The automated derivation of the action- and operator-dependent Feynman rules is performed in a separate step.
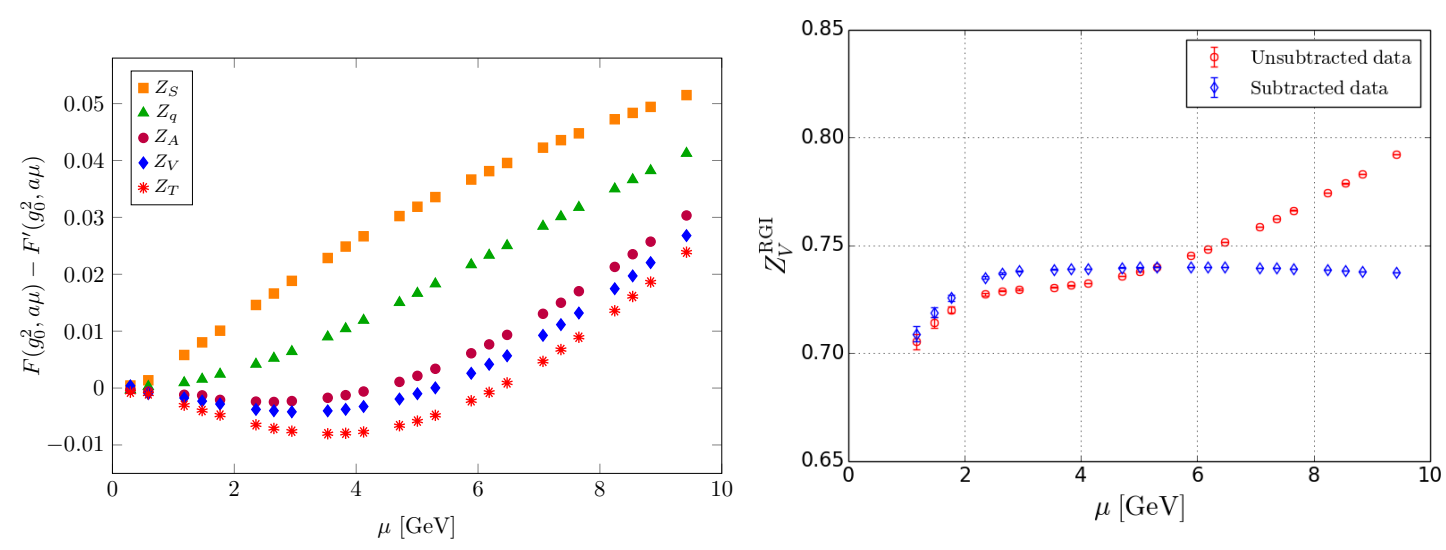

Figure 2: left: the subtraction functions $D(\mu, a)$ for a number of different renormalization constants; right: an example of an unsubtracted and the corresponding subtracted renormalization constant 
To combine the perturbative and non-perturbative results, we need to make a choice for the coupling. The bare lattice coupling is known to be generally a poor choice, and we employ the boosted coupling [11] instead. As can be seen from Fig. 2, this results in a cancellation of most of the lattice artifacts seen in the RGI renormalization constants.

\section{Results}

\subsection{Renormalization constants}

To obtain our final results for the RGI renormalization constants $Z^{\mathrm{RGI}}(\beta)$, we account for having performed the continuum matching only to three-loop order, and for the remaining lattice artifacts, by fitting with the ansatz

$$
Z^{\mathrm{RGI}, \mathrm{sub}}(a, \mu)=Z^{\mathrm{RGI}}(\beta)\left\{1+d_{1}\left[g^{\overline{\mathrm{MS}}}(\mu)\right]^{8}\right\}+d_{2}(\beta)(a \mu)^{2} \Delta Z^{\overline{\mathrm{MS}}}(\mu) Z_{\mathrm{RI}-\mathrm{MOM}}^{\overline{\mathrm{MS}}}(\mu),
$$

where $d_{1}$ is a continuum quantity and hence independent of the bare lattice coupling $\beta$, and we therefore perform a combined four-parameter fit accross all lattice spacings. Ideally, the fit window should fulfil $\Lambda^{\overline{\mathrm{MS}}} \ll \mu \ll a^{-1}$ to keep both higher-order perturbative effects and lattice artifacts small. Since we cannot realistically fulfil both of those inequalities at the same time, we have chosen to take the lower end of the window at $\mu_{\min }=3 \mathrm{GeV}$, but allow renormalization scales as large as $\mu_{\max }=2.75 a^{-1}$ in the fit, because we rely on the perturbative subtraction of the leading artifacts. The resulting fits are shown in Fig. 3.

To study the systematic errors of final result we have varied a number of procedure parameters:

- the form of the chiral extrapolation (quadratic instead of linear, or including a $\mathrm{e}^{-m_{\pi} L}$ finitevolume term),

- the value of $a \Lambda^{\overline{\mathrm{MS}}}$ used, and

- the fitting window for the final fit.

To be conservative, we add the spreads of the values obtained with each of these variations in quadrature to estimate our total systematic error. Preliminary values of the renormalization constants, including their systematic error estimates, are shown in Table 2.

Table 2: Preliminary results for the RGI renormalization constants of local bilinear operators; the errors shown are statistical and systematic (cf. the text for details on how systematics are estimated).

\begin{tabular}{cllll}
\hline \hline$\beta$ & $Z_{A}^{\text {RGI }}$ & $Z_{V}^{\text {RGI }}$ & $Z_{S}^{\text {RGI }}$ & $Z_{T}^{\text {RGI }}$ \\
\hline 5.2 & $0.7565(2)(7)$ & $0.7323(2)(5)$ & $0.473(2)(17)$ & $0.9036(2)(33)$ \\
5.3 & $0.7676(1)(10)$ & $0.7447(1)(22)$ & $0.469(2)(12)$ & $0.9192(1)(25)$ \\
5.5 & $0.7867(1)(23)$ & $0.7645(1)(50)$ & $0.459(1)(11)$ & $0.9500(1)(28)$ \\
\hline \hline
\end{tabular}



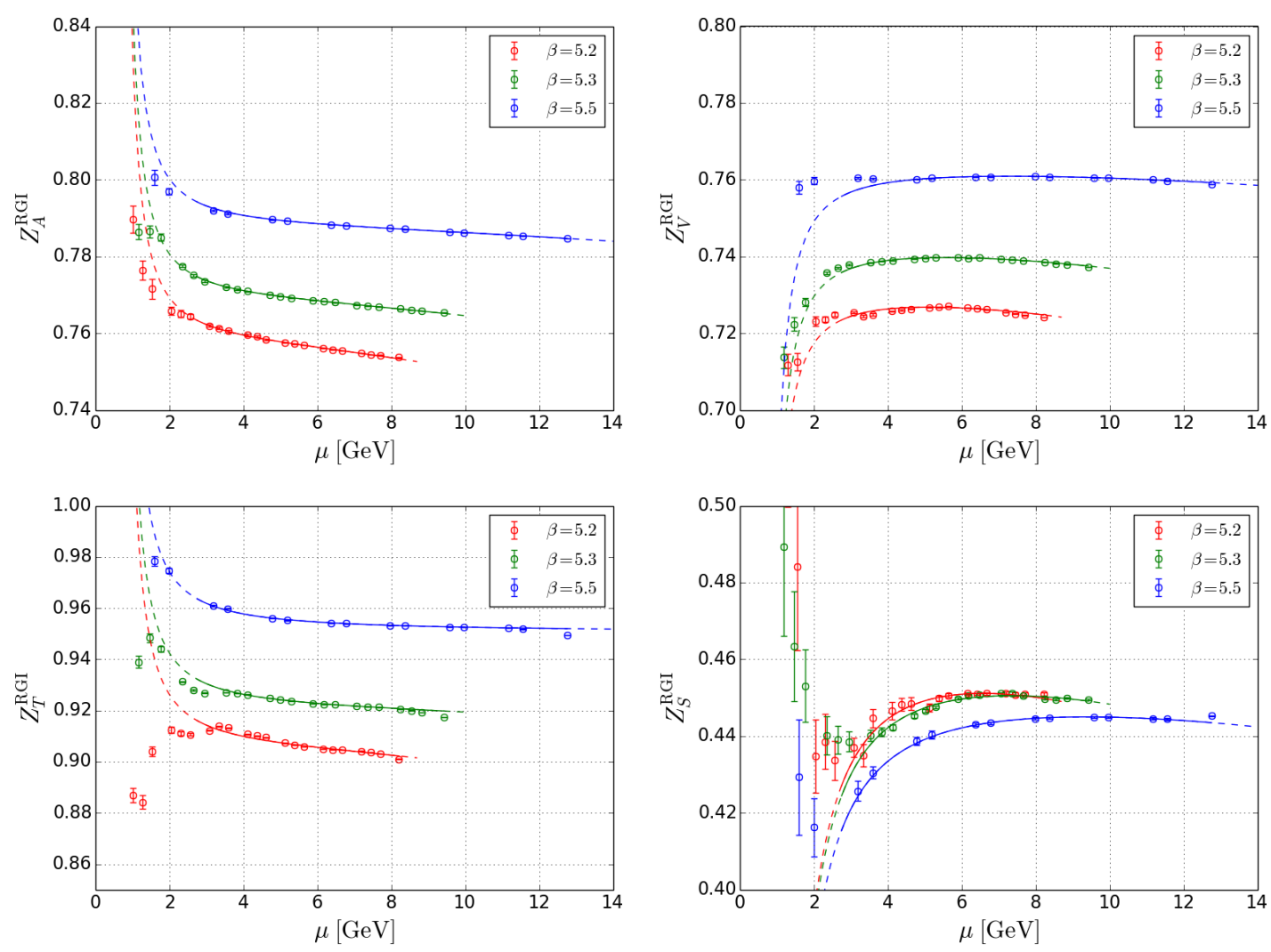

Figure 3: Final fits to the perturbatively subtracted RGI renormalization constants (clockwise from top left: axial, vector, scalar and tensor)

\subsection{Nucleon tensor charge}

As a sample application of our renormalization constants, we show some preliminary results for the nucleon tensor charge from the $N_{\mathrm{f}}=2$ CLS ensembles in Fig. 4. After being appropriately renormalized, the tensor charge shows no appreciable lattice-spacing dependence, but appears to be strongly pion-mass dependent, leading to a rather low value at the physical point.

A separate higher-statistics calculation [12] using many of the same ensembles, but employing different measurement and analysis techniques for the bare matrix elements, on the other hand, appears to show hardly any pion-mass dependence. However, we should caution that the latter analysis did not fully account for excited-state effects, and that a comprehensive analysis is still ongoing.

\section{Summary}

We have implemented non-perturbative renormalization for local quark bilinears using the RI'MOM scheme on the CLS $N_{\mathrm{f}}=2$ ensembles. The use of automated perturbation theory in carrying out the perturbative subtraction of lattice artifacts allows for an easy adaptation to different operators and actions. In particular, we intend to treat the derivative operators required for measuring $\langle x\rangle$ 

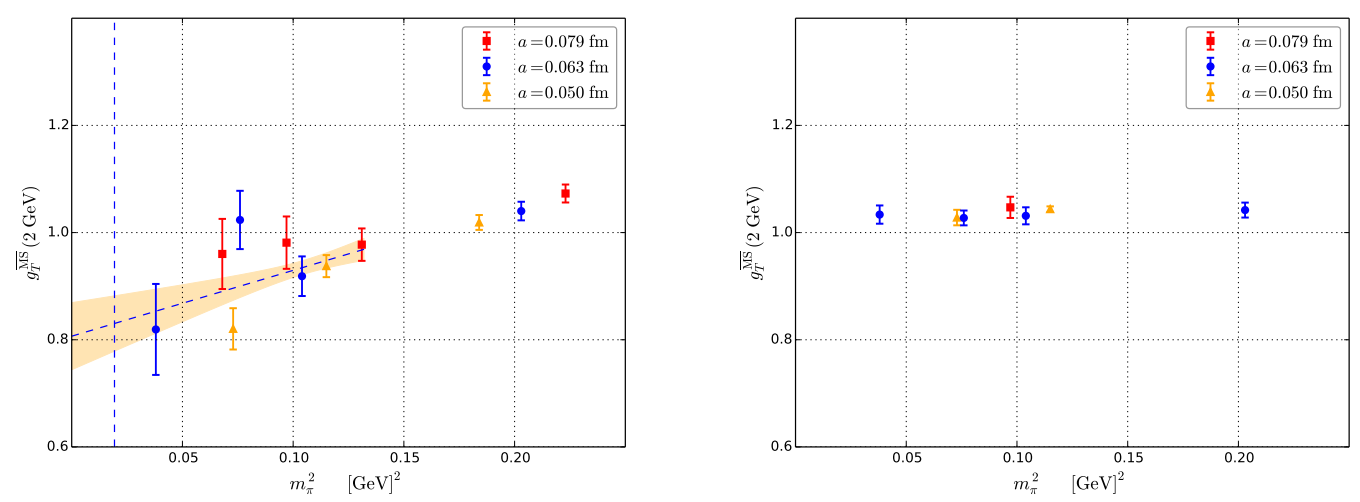

Figure 4: left: the tensor charge of the nucleon as a function of the pion mass, with a linear extrapolation to the physical point (preliminary); right: using higher statistics, the pion-mass dependence disappears (but see the text for caveats).

and related observables in much the same way. Moreover, the application to the $N_{\mathrm{f}}=2+1$ CLS ensembles (using the existing ensembles with periodic boundary conditions [13] for the NPR part) poses no major difficulties. Results for nucleon charges (and form factors) are forthcoming [14].

Acknowledgments: The authors acknowledge useful discussions with Meinulf Göckeler. We are grateful to our colleagues within the CLS initiative for sharing ensembles. Our calculations were performed on the "Clover" HPC Cluster at Helmholtz Institute Mainz; we are grateful to Dalibor Djukanovic for technical support.

\section{References}

[1] G. Martinelli et al., Nucl. Phys. B445 (1995) 81.

[2] M. Göckeler et al., Nucl. Phys. B544 (1999) 699.

[3] R. J. Hudspith, Comput. Phys. Commun. 187 (2015) 115.

[4] M. Göckeler et al., Phys. Rev. D82 (2010) 114511.

[5] J. A. Gracey, Nucl. Phys. B662 (2003) 247.

[6] T. van Ritbergen, J. A. M. Vermaseren and S. A. Larin, Phys. Lett. B400 (1997) 379.

[7] D. Becirevic et al., JHEP 0408 (2004) 022.

[8] M. Constantinou et al., Phys. Rev. D87 (2013) 096019.

[9] J. Simeth et al., PoS(LATTICE2014)294.

[10] A. Hart et al., Comput. Phys. Comm. 180 (2009) 2698.

[11] G. P. Lepage and P. B. Mackenzie, Phys. Rev. D48 (1993) 2250.

[12] G. von Hippel et al., Nucl. Phys. B914 (2017) 138.

[13] G. S. Bali et al., Phys. Rev. D94 (2016) 074501.

[14] D. Djukanovic et al., in preparation. 